\title{
In vitro controlled drug release from loaded microspheres - dose regulation through formulation
}

\author{
Laura J. Waters and Evangelos V. Pavlakis \\ Division of Pharmacy and Pharmaceutical Sciences, University of Huddersfield, Queensgate, Huddersfield, UK
}

Received August 6, 2007; Accepted September 26, 2007; Published October 8, 2007.

\begin{abstract}
Purpose. Drug release profiles were established for ibuprofen encapsulated within several types of microspheres and a range of dissolution buffer media to study the effect these variables have in controlling the rate and extent of drug release. Methods. Fatty acid microspheres containing ibuprofen were prepared by a process previously developed and refined to produce microspheres of a known size and composition, namely $80-125 \mu \mathrm{m}$ diameter and an excipient to ibuprofen ratio of 3:1. Drug release profiles from these encapsulated formulations were compared with those obtained for the dissolution of ibuprofen alone under the same conditions. Results. Stearic acid microspheres were found to only partially retard the release of ibuprofen over a twenty minute period compared with the dissolution of ibuprofen alone. However, a significant retardation of ibuprofen release was observed with cetostearyl alcohol microspheres over the same period of time. Secondly, drug release profiles for encapsulated ibuprofen were determined using five distinct dissolution buffer media; sodium phosphate, potassium phosphate, citric acid and phosphate mix, MOPS and tris. Significant differences in the extent and rate of drug release were recorded between the different dissolution buffer solutions. These differences were also shown to be independent of variations in $\mathrm{pH}$, temperature, buffer concentrations and the type of cations present. Conclusions. The presence and choice of microsphere formulation, and the choice of buffer present in the dissolution solution, can influence drug release in vitro, i.e. it is possible to achieve controlled drug release from microspheres. To explain the control achieved through the choice of buffer in solution it is proposed that the buffer anion exerts a stabilising influence on the ibuprofen-microsphere matrix.
\end{abstract}

\section{NOVELTY OF THE WORK}

The research presented in this paper introduces a new formulation for pharmaceutical applications, namely encapsulating a drug within stearic acid and cetostearyl alcohol microspheres. Several interesting observations were made in that different microsphere formulations retarded drug release in vitro to differing degrees and surprisingly, the buffer anion appeared to dictate drug release. It is therefore now possible to produce a unique formulation with a highly controlled level of drug release, i.e. achieving dose regulation through formulation.

\section{INTRODUCTION}

Ibuprofen $\quad[( \pm)-2-(4$ '-isobutylphenyl)propanoic acid] is a non-steroidal anti - inflammatory drug (NSAID), which also exhibits antipyretic and analgesic properties (1). Ibuprofen is available in a wide range of formulations, the most popular preparation being the tablet dosage form. However, it is difficult to formulate the drug as either an aqueous solution or as a syrup because of the physicochemical properties of the drug. The low solubility of ibuprofen in a suitable medium is responsible for the most severe limiting factor in formulation development. An alternative way of delivering the drug is to use microencapsulation technology (2) which allows the drug to be trapped in a small sphere with a diameter ranging from $1-1500 \mu \mathrm{m}(3,4)$. The resultant product consists of monolithic spherical structures containing a uniform dispersion of the drug (5). Careful consideration of the composition of the encapsulation medium not only improves drug solubility, but may also facilitate targeted and regulated drug delivery (6) through controlling the rate of drug release, and hence absorption of the drug in vivo (7).

A material that has recently found application as an encapsulation medium is stearic acid (8) which is inert, non - toxic, cheap and listed as an acceptable excipient in the the United States Pharmacopeia (USP).

Corresponding Author: Dr Laura Waters, Division of Pharmacy and Pharmaceutical Sciences, University of Huddersfield, Queensgate, Huddersfield, UK; E-mail: l.waters@hud.ac.uk 
The use of stearic acid microspheres may also improve patient compliance in that they could provide a taste-masking effect for medications possessing an unpleasant taste (9). Hitherto, only a small amount of research using drug incorporation within stearic acid microspheres has been carried out, for instance using the antibiotic cefuroxime axetil (10-12). Microspheres to encapsulate drugs can, of course, be synthesised from alternatives to stearic acid (13). For example ibuprofen has previously been incorporated within ceto-stearyl alcohol microspheres (14) but to date, drug release profiles based on dissolution studies have not been fully investigated.

Certain factors are known to affect drug release measurements; these include the $\mathrm{pH}(15)$, temperature (16) and ionic strength (17) of the dissolution medium, the dissolution method chosen (18) - including the presence and thickness of an unstirred layer, and the presence of various coexcipients (19). Additionally, it has been demonstrated that the dissolution medium buffer composition can play a role in the rate and extent of drug release (20). This is the case for the ibuprofen-related drug, ketoprofen (21). Buffer composition can have a significant effect on solubility of a drug and it has been found that ibuprofen release does vary, although the rates do not appear to have any correlation with the solubility of the salt formed (22).

In this work a comparative analysis for free and encapsulated ibuprofen is presented. Microspheres of stearic acid, cetostearyl alcohol and a combination of the two are the chosen encapsulation media from which the drug is released into a range of buffered solutions. The objective of this work is to establish which factors retard or enhance the rate and extent of ibuprofen release from the microsphere-based formulations. By understanding these relationships, it may be possible in the future to design more tailored formulations with more desirable drug release profiles than those currently available.

\section{METHODS}

\section{Materials}

Stearic acid $\left(\mathrm{CH}_{3}\left(\mathrm{CH}_{2}\right)_{16} \mathrm{COOH}\right)$ was purchased from Fluka (Switzerland). Ibuprofen and cetostearyl alcohol (a mixture of $\mathrm{CH}_{3}\left(\mathrm{CH}_{2}\right)_{15} \mathrm{OH}$ and $\left.\mathrm{CH}_{3}\left(\mathrm{CH}_{2}\right)_{17} \mathrm{OH}\right)$ were provided by Thornton and Ross Ltd. (Huddersfield, UK). Sodium phosphate, potassium phosphate, citric acid, MOPS $\left(\mathrm{C}_{7} \mathrm{H}_{15} \mathrm{NO}_{4} \mathrm{~S}\right)$, MOPS sodium salt $\left(\mathrm{C}_{7} \mathrm{H}_{14} \mathrm{NO}_{4} \mathrm{SNa}\right)$ and tris $\left(\mathrm{NH}_{2} \mathrm{C}\left(\mathrm{CH}_{2} \mathrm{OH}\right)_{3}\right)$ were purchased from Sigma-Aldrich (Dorset, UK). All reagents used were within a purity range from $95 \%$ to $99.5 \%$.

\section{Ibuprofen-loaded microsphere preparation}

The method adopted for microsphere preparation in this work is similar to that previously reported (23) with minor alterations as detailed below.Stearic acid or cetostearyl alcohol and ibuprofen, weighed out at a 3:1 mass ratio (with a total mass of $100 \mathrm{~g}$ ), were melted in a pre-heated $\left(85^{\circ} \mathrm{C}\right)$ reaction vessel. De-ionised water $(1 \mathrm{~L})$ was heated to $85^{\circ} \mathrm{C}$ on a hot - plate and added to the molten mixture. This was then left for 5 minutes at $85^{\circ} \mathrm{C}$ and stirring then commenced. Experiments were conducted with a stirring speed of $3000 \mathrm{rpm}$. After 5 minutes, the heating element of the water bath was switched off, allowing the temperature of the system to fall. Cold water $\left(5^{\circ} \mathrm{C}\right)$ was circulated around the system, cooling down the resulting emulsion in less than 15 minutes. When the temperature of the system reached $30^{\circ} \mathrm{C}$, the stirrer was switched off and the resulting microspheres collected by suction filtration using a Büchner filter. The solid product collected was dried overnight in a vacuum desiccator over silica gel. The microspheres were sieved and those with a diameter between $80 \mu \mathrm{m}$ and $125 \mu \mathrm{m}$ collected and used for all subsequent studies.

This methodology was adopted since it produces microspheres of a known maximum diameter $(125 \mu \mathrm{m})$ and containing a fixed amount of ibuprofen (it was reasonably assumed that all of the added ibuprofen was incorporated within the microspheres). The process is also fast, relatively economical and safe, since no flammable or toxic organic solvents are required during production. Moreover, the formulation process did not appear to affect the integrity of the ibuprofen samples in any way.

\section{Particle Size}

The particle size was determined using a laser diffractometer (Malvern Mastersizer 2000, UK). Six aqueous solutions of ibuprofen (each $0.08 \% \mathrm{vol}$ ) were measured and the overall mean particle size recorded. 


\section{Scanning Electron Microspcopy}

Scanning electron microscopy (SEM) studies were conducted on microspheres containing ibuprofen after exposure to a series of buffer solutions to observe surface changes to the microspheres. All samples were coated with a gold/palladium mixture for 2 minutes at $18 \mathrm{~mA}$ using a sputter coater to achieve a layer a few atoms thick. Coated samples were examined using a Jeol (JSM-6060 LV) microscope at an accelerating voltage of $2.0 \mathrm{kV}$.

\section{Dissolution Testing}

Dissolution studies were performed using an automated assembly, comprising a dissolution bath (Pharmatest PTW III) and a UV visible spectrophotometer (Cecil 3021, series 3000).

For the first series of experiments either 125 $\mathrm{mg}$ of ibuprofen or weighed quantities of microspheres containing $125 \mathrm{mg}$ of ibuprofen were placed in six separate 1 litre containers containing aqueous phosphate buffer $(0.2 \mathrm{M}$ monobasic and $0.2 \mathrm{M}$ dibasic sodium phosphate mixed together to produce a solution with $\mathrm{pH} 8$ ). For the second series of experiments, dissolution media comprised of five separate buffer solutions, all prepared at $\mathrm{pH} 8$, incorporating a range of cations and anions. The buffers consisted of the following pairs of compounds titrated together to achieve $\mathrm{pH} 8$ solutions at $37^{\circ} \mathrm{C}: 0.2 \mathrm{M}$ monobasic and $0.2 \mathrm{M}$ dibasic sodium phosphate $\left(\mathrm{NaH}_{2} \mathrm{PO}_{4}\right.$ and $\left.\mathrm{Na}_{2} \mathrm{HPO}_{4}\right), 0.2 \mathrm{M}$ monobasic and $0.2 \mathrm{M}$ dibasic potassium phosphate $\left(\mathrm{KH}_{2} \mathrm{PO}_{4}\right.$ and $\left.\mathrm{K}_{2} \mathrm{HPO}_{4}\right), 0.1 \mathrm{M}$ citric acid and $0.2 \mathrm{M}$ dibasic sodium phosphate $\left(\mathrm{C}_{6} \mathrm{H}_{8} \mathrm{O}_{7}\right.$ and $\left.\mathrm{Na}_{2} \mathrm{HPO}_{4}\right), 0.1 \mathrm{M}$ MOPS and $0.1 \mathrm{M}$ MOPS sodium salt $\left(\mathrm{C}_{7} \mathrm{H}_{15} \mathrm{NO}_{4} \mathrm{~S}\right.$ and $\left.\mathrm{C}_{7} \mathrm{H}_{14} \mathrm{NO}_{4} \mathrm{SNa}\right)$ and finally, 0.1 $\mathrm{M}$ tris and $0.1 \mathrm{M}$ hydrochloric acid $\left(\mathrm{NH}_{2} \mathrm{C}\left(\mathrm{CH}_{2} \mathrm{OH}\right)_{3}\right.$ and $\left.\mathrm{HCl}\right)$. Finally, for the third series of experiments, drug release studies were conducted for ibuprofen contained within stearic acid microspheres using a sodium phosphate buffer over a range of sodium and phosphate concentrations $(0.01 \mathrm{M}$ to $0.8 \mathrm{M}$ and $0.02 \mathrm{M}$ to $0.2 \mathrm{M}$ respectively).

During all dissolution testing a constant stirring speed and temperature were maintained at $200 \mathrm{rpm}$ and $37^{\circ} \mathrm{C}$ respectively. Changes in the absorbance values at $235 \mathrm{~nm}$ were recorded for 20 minutes at two minute intervals. Results were replicated $(n=6)$ for statistical analysis and a mean value for absorbance was then calculated to obtain the percentage of ibuprofen released for each time interval. It should be noted that prior to drug release experiments it was found that the presence of ibuprofen and/or microspheres did not affect the $\mathrm{pH}$ of the dissolution media under any conditions investigated in this research. A solution of $\mathrm{pH} 8$ was chosen in order to optimise the solubility of the ibuprofen.

\section{RESULTS}

\section{Effect of microsphere composition on the release of ibuprofen}

The effect of microsphere composition on the release of ibuprofen is shown in Figure 1, where the percentage of drug released into phosphate buffer is plotted as a function of time. For comparative studies, ibuprofen (with a mean diameter of $53.6 \mu \mathrm{m}$, as determined by particle size analysis) was also employed. It should be noted that the profile displayed for ibuprofen alone in Figure 1 is correctly described as a dissolution profile rather than a release profile as there is no microsphere for the drug to release from.

From Figure 1 differences in the dissolution profile for ibuprofen powder alone compared with release profiles for the three microsphere formulations are evident proving that drug release can effectively be controlled by the incorporation of ibuprofen within microspheres of different compositions. Figure 1 also clearly shows that cetostearyl alcohol microspheres most significantly retard the release of ibuprofen over a twenty minute period compared with ibuprofen alone with a final percentage difference of $79 \%$. Direct comparison of the four release profiles in Figure 1 shows that three appear asymptotic yet the fourth (cetostearyl alcohol microspheres) appears linear. Therefore, the physicochemical mechanism of drug release from this fourth formulation is distinctly different from the others although the reason for this difference is unknown at this time.

\section{Effect of dissolution media composition on the dissolution of ibuprofen}

The effect of buffer media composition on the dissolution of ibuprofen powder alone is shown in Figure 2, where the percentage of drug 'released' into aqueous solutions of five different buffer solutions is plotted as a function of time. 


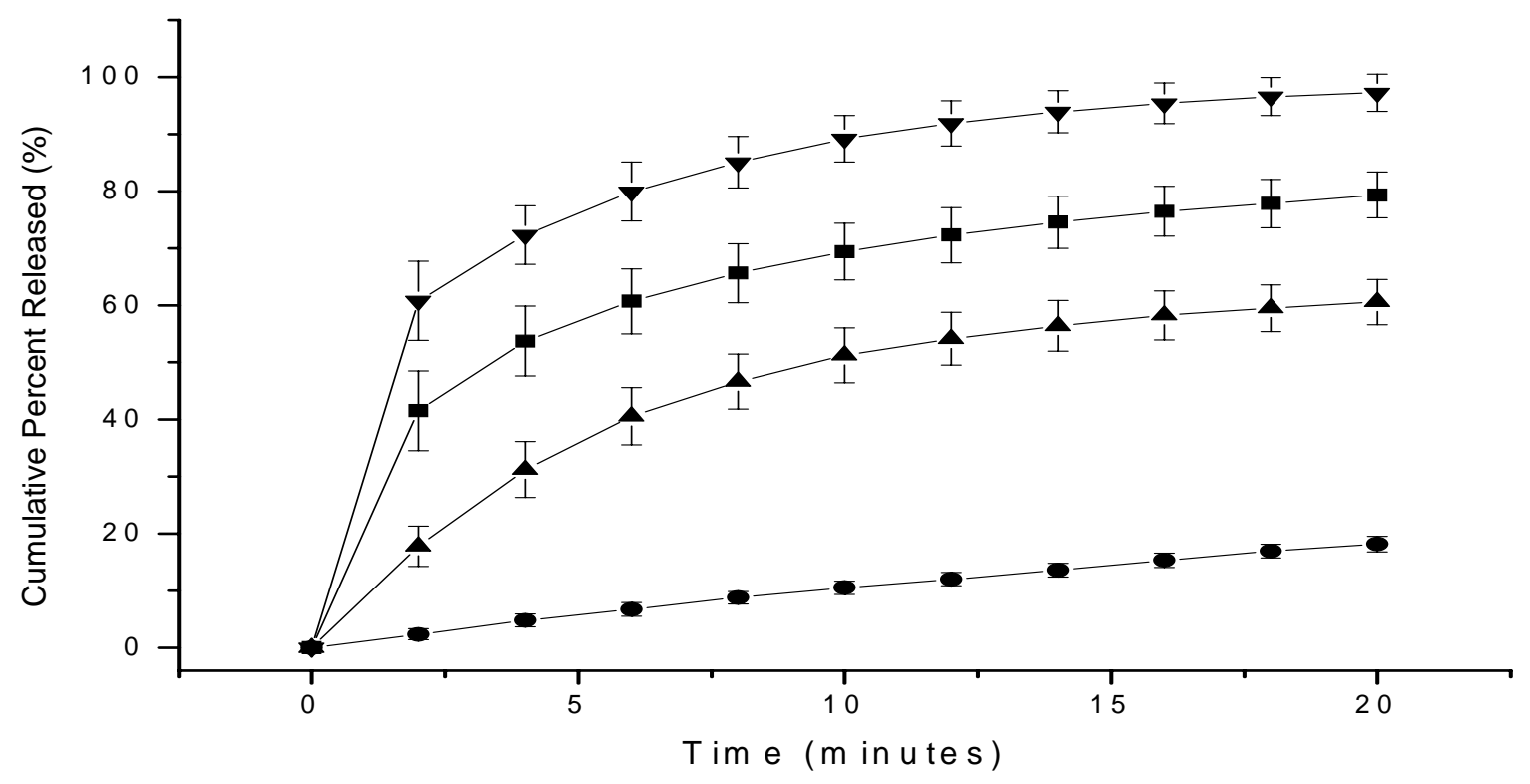

Figure 1. Dissolution profile at $37^{\circ} \mathrm{C}$ in $\mathrm{pH} 8$ phosphate buffer for ibuprofen powder alone $(\boldsymbol{\nabla})$, release profiles of ibuprofen from: stearic acid microspheres $(\boldsymbol{\bullet}), 1: 1$ ratio of stearic acid and cetostearyl alcohol microspheres $(\boldsymbol{\Lambda})$ and cetostearyl alcohol microspheres $(\bullet)$. Each data point represents the mean of 6 results with SD error bars.

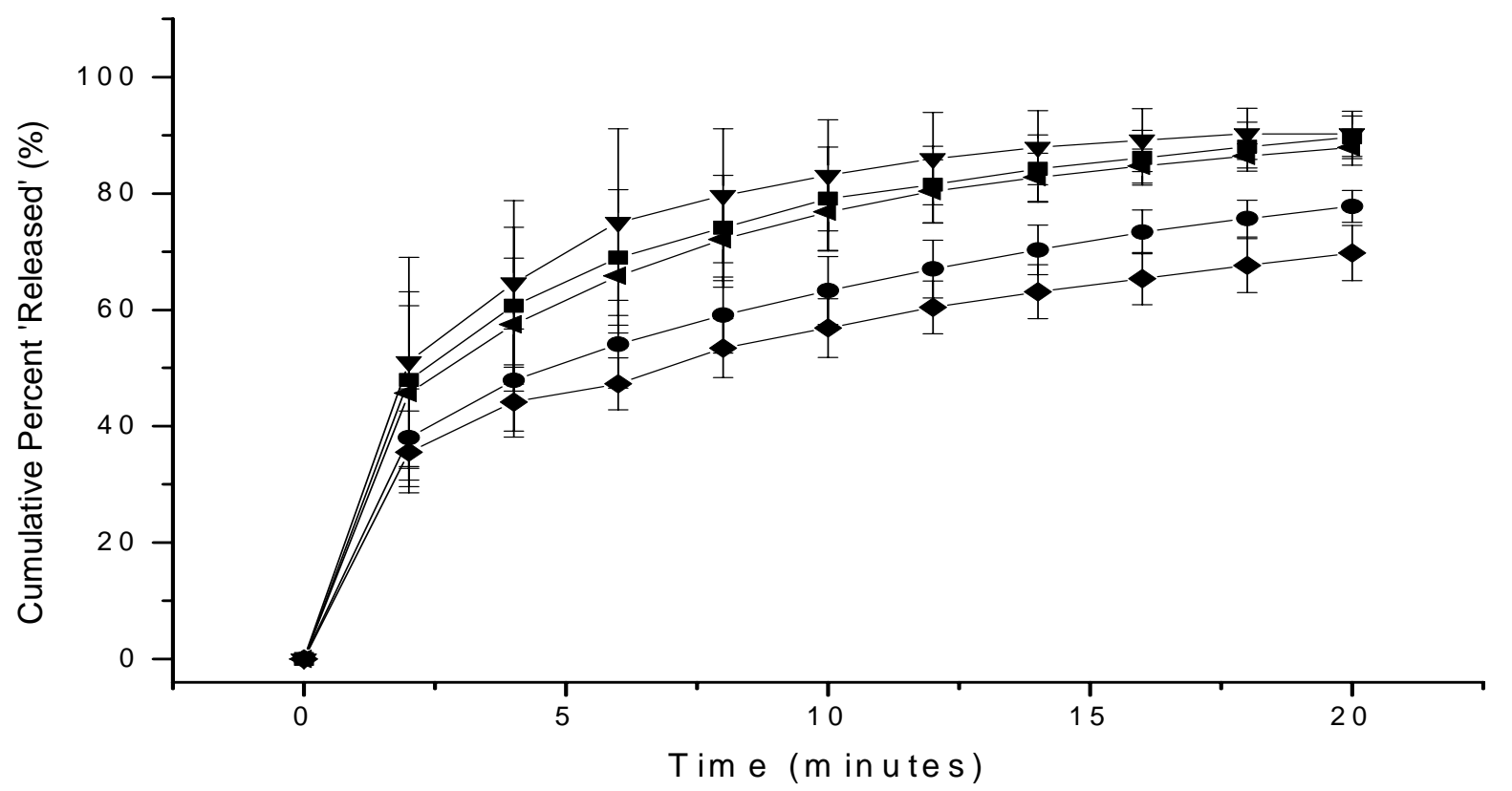

Figure 2. Dissolution profiles of ibuprofen at $37^{\circ} \mathrm{C}$ in five $\mathrm{pH} 8$ buffer solutions: $\mathrm{NaH}_{2} \mathrm{PO}_{4}$ and $\mathrm{Na}_{2} \mathrm{HPO}_{4}(\varangle)$, $\mathrm{KH}_{2} \mathrm{PO}_{4}$ and $\mathrm{K}_{2} \mathrm{HPO}_{4}(\bullet), \mathrm{C}_{6} \mathrm{H}_{8} \mathrm{O}_{7}$ and $\mathrm{Na}_{2} \mathrm{HPO}_{4}(\boldsymbol{\nabla})$, MOPS $(\bullet)$ and tris $(\bullet)$. Each data point represents the mean of 6 results with $\mathrm{SD}$ error bars. 
From the results in Figure 2 differences in the dissolution profiles for ibuprofen in the five buffer solutions are again apparent. Three buffers produced similar final percentages of drug 'release', namely the combined citric acid and phosphate buffer, the potassium phosphate buffer and the sodium phosphate buffer. The two remaining buffers produced a slightly slower dissolution rate and significantly lower final percentage values.

The effect of buffer composition on the release of ibuprofen from stearic acid microspheres is shown in Figure 3. For these experiments, stearic acid microspheres were chosen in particular to represent an encapsulated formulation for comparison with Figure 2. All results displayed in Figures 2 and 3 were obtained using a pH 8 buffered aqueous solution.

Figure 3 displays significant differences in the release profiles for ibuprofen from stearic acid microspheres for the five buffer solutions. Overall, there are two distinct categories of release, those from phosphate based buffer solutions and the remaining three buffer solutions. The percentage drug release difference between the highest and lowest final percentage release was significant at $62 \%$ indicating that the choice of buffer used can dramatically influence release rates for the ibuprofen-stearic acid system.

Comparison between Figures 2 and 3 provides two interesting observations. Firstly, the rate of ibuprofen dissolution/release is the same for two of the buffers for ibuprofen powder alone and for ibuprofen from stearic acid microspheres, namely the sodium phosphate and the potassium phosphate buffer systems. In marked contrast, the rates of dissoluton/release were very different for the ibuprofen powder alone and the encapsulated drug in the three remaining buffer systems. For example, using the citric acid and phosphate buffer the encapsulated ibuprofen released $41 \%$ less after twenty minutes than the ibuprofen powder alone. A similar relationship can be seen for the two other buffers considered, with tris and MOPs buffers releasing 39 and $31 \%$ less ibuprofen respectively from the microspheres compared with ibuprofen powder alone.

Alongside dissolution studies, scanning electron microscopy (SEM) was used to observe surface changes to the microspheres upon immersion in a series of buffers. Formulated microspheres containing ibuprofen were compared after exposure to $\mathrm{pH} 8$ buffer solutions for 10 minutes each at $37^{\circ} \mathrm{C}$. SEM images are shown in Figure 4.

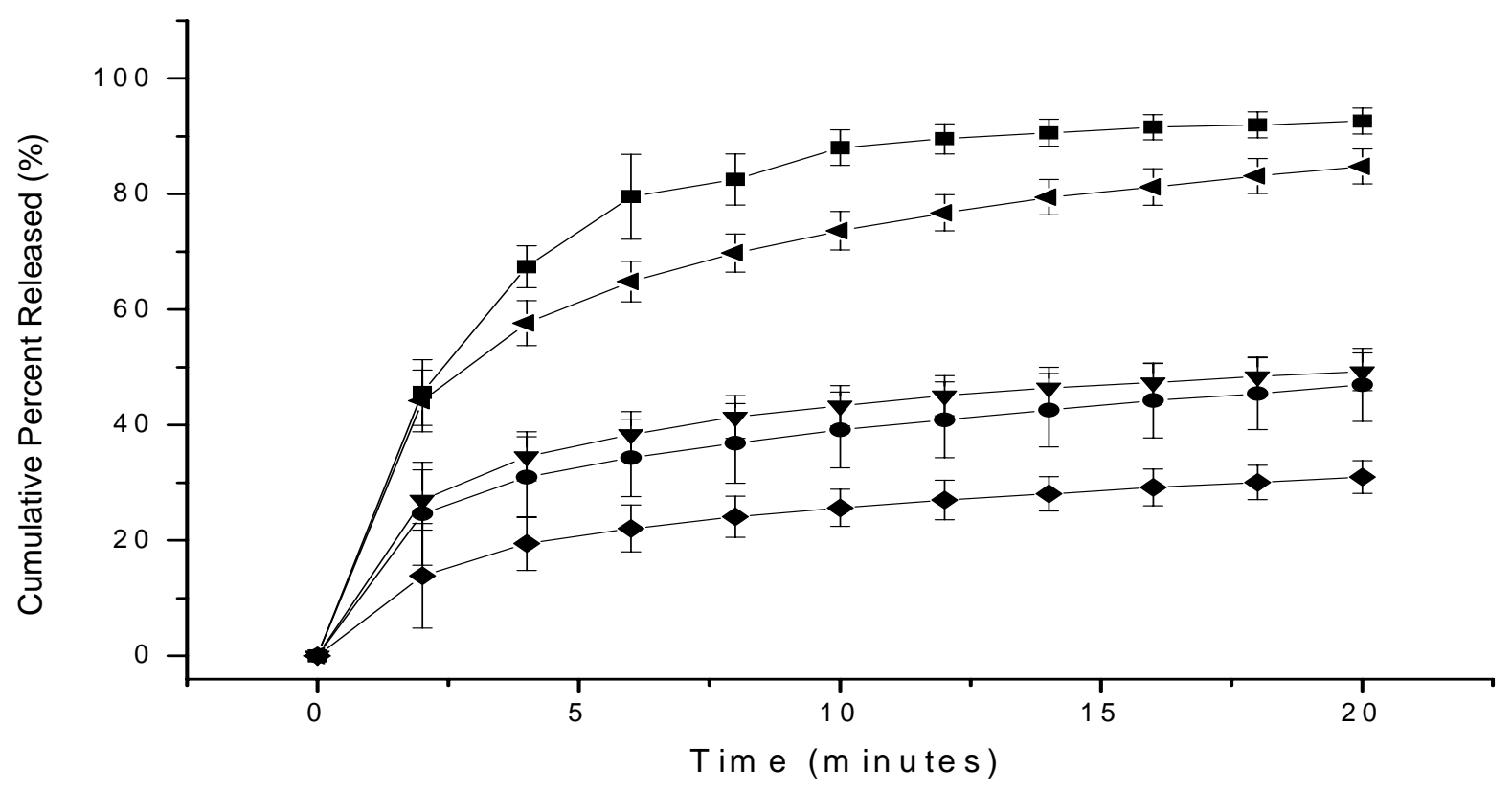

Figure 3. Release profiles of ibuprofen from stearic acid microspheres at $37^{\circ} \mathrm{C}$ in five pH 8 buffer solutions: $\mathrm{NaH}_{2} \mathrm{PO}_{4}$ and $\mathrm{Na}_{2} \mathrm{HPO}_{4}(\bullet), \mathrm{KH}_{2} \mathrm{PO}_{4}$ and $\mathrm{K}_{2} \mathrm{HPO}_{4}(\boldsymbol{\bullet}), \mathrm{C}_{6} \mathrm{H}_{8} \mathrm{O}_{7}$ and $\mathrm{Na}_{2} \mathrm{HPO}_{4}(\boldsymbol{\nabla})$, MOPS $(\bullet)$ and tris $(\diamond)$. Each data point represents the mean of 6 results with $\mathrm{SD}$ error bars. 


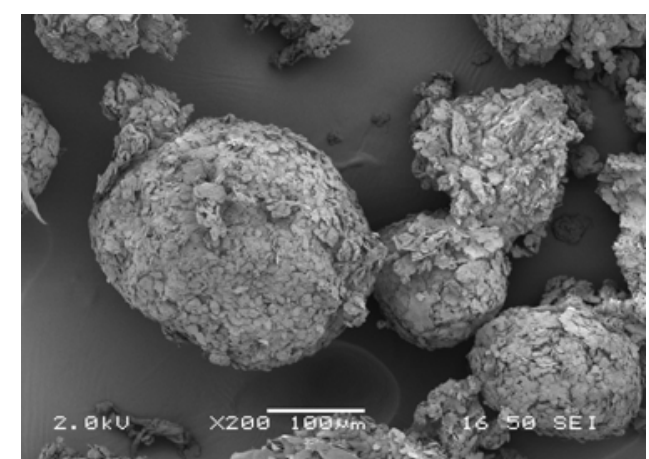

(a)

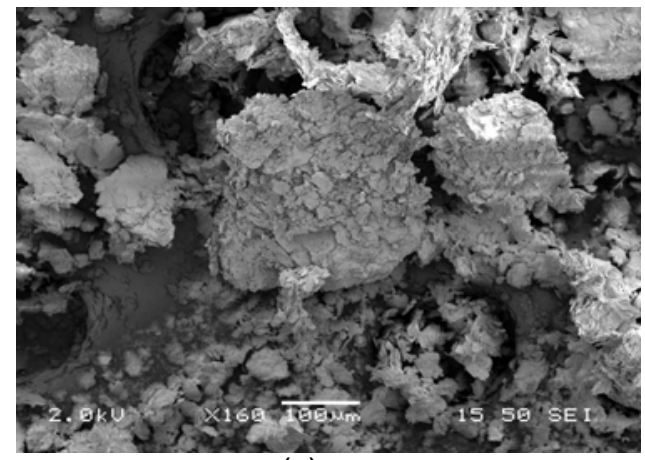

(c)

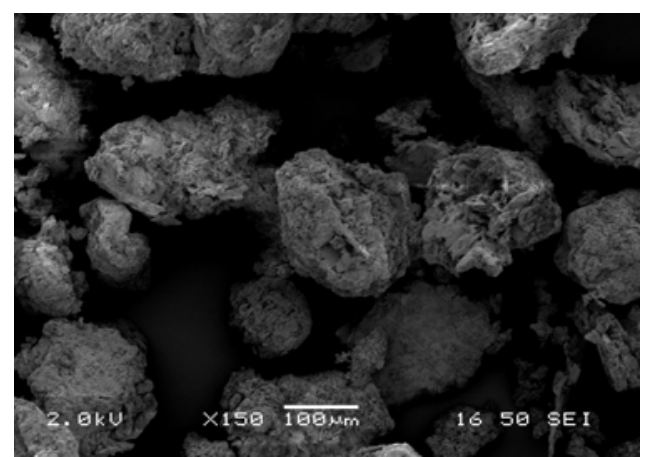

(b)

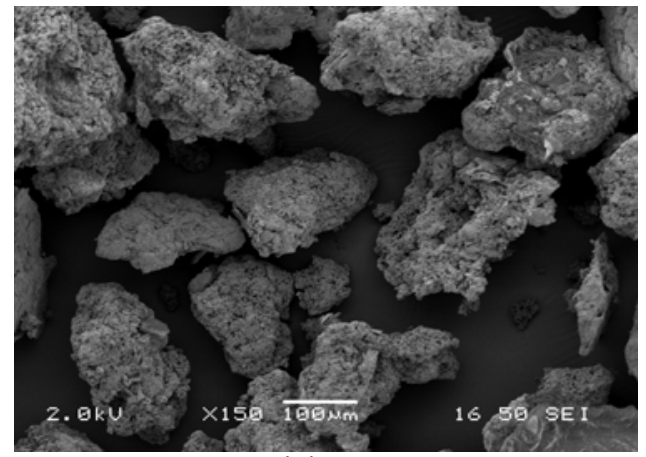

(d)

Figure 4. SEM images for the stearic acid microspheres after immersion in $\mathrm{pH} 8$ buffer at $37^{\circ} \mathrm{C}$ : sodium phosphate buffer (a), potassium phosphate buffer (b), MOPS buffer (c) and tris buffer (d).

From the images displayed in Figure 4 it can be seen that the microspheres have undergone morphological changes following immersion in each of the buffer solutions. Firstly, upon immersion in sodium phosphate buffer the microspheres have appeared to swell with a rougher surface yet still retaining their spherical morphology. The remaining three images appear to show no significant size change although the microspheres are less spherical, more angular and in particular, following immersion in MOPS buffer solution, rather fragmented.

\section{DISCUSSION}

Results presented in this paper provide two distinct findings of relevance to assist intentionally controlling drug release from microspheres. Firstly, results for the profiles for the release of ibuprofen from stearic acid microspheres compared with cetostearyl alcohol microspheres show that drug release can be effectively retarded through formulation choice over the period of time under investigation in this study. Previous consideration of systems similar to those investigated here provide equations to assist the understanding of the physicochemical drug release mechanisms involved (24). From these equations, and data presented in Figure 1, it is possible to determine for each scenario if drug release is diffusion controlled, anomalous diffusion (i.e. non-Fickian) or zero order. Drug release for the stearic acid formulation was found to be non-Fickian whereas for the cetostearyl alcohol formulation diffusion was the main release mechanism. Interestingly, the combined formulation of the two was found to have diffusion and matrix relaxation playing an equal role, i.e. displaying zero order drug release.

Secondly, the nature of the buffer electrolyte has a major effect on microsphere morphology (SEM results) and ibuprofen release (dissolution/release profile results). Since dependence on the rate of drug release is greater for the encapsulated ibuprofen it is possible that there is some form of interaction between the buffer and the stearic acid itself. However, this has been postulated previously and was thought 
to be connected to the presence of the buffer cation associated with the stearate anion which could exchange with that from the buffer solution (11). A modified version of this accepted theory is to consider that the cation may also simultaneously experience an interaction with a surrounding buffer anion thus creating a 'protected' environment and preventing microsphere disintegration with consequent reductions in ibuprofen release (Figure 5). This proposed model is best described as a charged double layer, similar to that routinely used to explain the stability of colloidal dispersions.

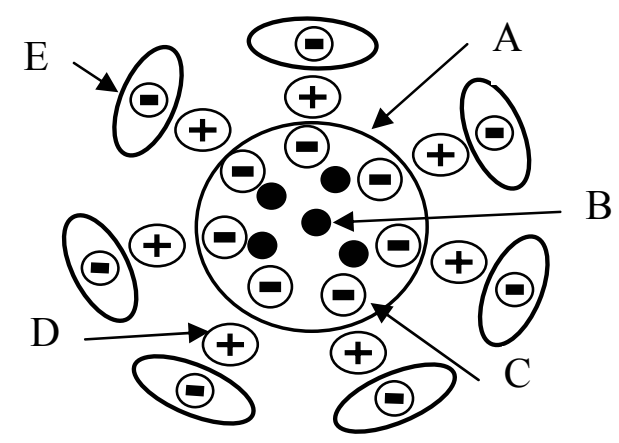

Figure 5. Diagrammatic representation to illustrate the proposed 'buffer anion protection' theory with the microsphere (A), including embedded drug (B), with exposed stearate anions (C), surrounded by buffer cations (D) and finally protected by buffer anions (E).

In both Figures 2 and 3 the results clearly show that tris displays the lowest rate of drug release which may be ascribed to the fact that it is not present as an anion thus there is no chargestabilising effect. Previous authors have noticed that some buffers can act as surface active agents with an apparent increase in drug release (21). Although in theory this could possibly be true for two of the buffers considered here, i.e. MOPS and tris, the results suggest that this is not the case. There is no observable increase in the rate or extent of drug release for ibuprofen during the course of the experiment with these buffers.

The results also prove that this does not appear to occur with phosphate anions. At this stage, the mechanism for the selective stabilising effects of the buffers is speculative. However, it may be that stabilisation is related to the size and therefore the nucleophilicity of the buffer anion. The phosphate anion is much smaller than the others, and there may be a minimum anion size required to stabilise the stearic acid microspheres. This hypothesis is based on an assumption that buffer capacity does not play a significant role in the results obtained. However, to eliminate any concerns that existed regarding the potential variability of buffer concentration, and therefore buffer capacity, a final set of experiments was conducted.

\section{Effect of buffer concentration on the release of ibuprofen}

As previously mentioned, it was imperative to determine whether or not the buffer cation and anion concentrations had any bearing on the drug release before conclusions could truly be drawn on the observations reported earlier in this work. The effect of a series of concentrations of sodium phosphate buffer $(0.02 \mathrm{M}$ to $0.2 \mathrm{M})$ on the release of ibuprofen from stearic acid microspheres over a twenty minute period was investigated. The results obtained show that no statistically significant differences were observed in the total percentage of drug release. This also supports the view that phosphate, unlike the other buffers, has no apparent stabilising or destabilising effect on the microspheres.

To ensure no effect was observed through variation in sodium ion concentration, a similar set of experiments was also conducted with the addition of a series of concentrations of sodium chloride $(0.01 \mathrm{M}$ to $0.8 \mathrm{M})$. Again, no statistically significant difference in the total percentage of drug release was observed over a 20 minute period.

\section{CONCLUSION}

In conclusion, the presence and choice of microsphere formulation, and the choice of buffer, influenced both the rate and extent of ibuprofen release into solution, i.e. by regulating certain parameters it is possible to achieve solution controlled drug release from microspheres. Cetostearyl alcohol microspheres significantly retarded ibuprofen release into solution compared with either stearic acid microspheres or a mixture of the two. A clear effect on microsphere morphology and the dissolution profile was also seen in the presence of the buffer solutions. Data suggests that there is a connection between buffer anion choice and drug release and it is proposed that this is because the anion exerts a stabilising influence on the ibuprofen-microsphere matrix. Therefore, having determined factors which permit a new degree of control over drug release it will, in theory, be 
possible to develop more tailored formulations in the future.

\section{ACKNOWLEDGMENTS}

Authors gratefully acknowledge financial support received from the University of Huddersfield, UK.

\section{REFERENCES}

[1]. C. Baum, D.L. Kennedy, M.B. Forbes, Utilization of nonsteroidal antiinflammatory drugs. Arthritis Rheum., 28:686 - 692, 1985.

[2]. M.E. Morales, V. Gallardo Lara, A.C. Calpena, J. Doménech, M.A. Ruiz, Comparative study of morphine diffusion from sustained release polymeric suspensions. J. Control. Release, 95: 75-81, 2004.

[3]. C. Dubernet, Thermoanalysis of microspheres. Thermochim. Acta, 248:259 - 269, 1995.

[4]. L. Choisnard, A. Géze, M. Bigan, J. Putaux, D. Wouessidjewe, Efficient size control of ampiphilic cyclodextrin nanoparticles through a statistical mixture design methodology. J Pharm Pharm Sci., 8(3):593-600, 2005.

[5]. W.H. Liu, J.L. Song, K. Liu, D.F. Chu, Y.X. $\mathrm{Li}$, Preparation and in vitro and in vivo release studies of Huperzine A loaded microspheres for the treatment of Alzheimer's disease. J. Control. Release, 107:417-427, 2005.

[6]. M. Momin, K. Pundarikakshudu, In vitro studies on guar gum based formulation for the colon targeted delivery of Sennosides. J Pharm Pharm Sci, 7(3):325-331, 2004.

[7]. R.H. Müller, S. Runge, V. Ravelli, W. Mehnert, A.F. Thünemann, E.B. Souto, Oral bioavailability of cyclosporine: Solid lipid nanoparticles $\left(\mathrm{SLN}^{\circledR}\right)$ versus drug nanocrystals. Int. J. Pharm., 317: 82-89, 2006.

[8]. A. Lavasanifar, J. Samuel, G.S. Kwon, The effect of fatty acid substitution on the in vitro release of amphotericin B from micelles composed of poly(ethylene oxide)-blockpoly(N-hexyl stearate-L-aspartamide). J. Control. Release, 79:165-172, 2002.

[9]. G. Wei $\beta$, A. Knoch, A. Laicher, F. Stanislaus, R. Daniels, Simple coacervation of hydroxypropyl methylcellulose phthalate (HPMCP) II. Microencapsulation of ibuprofen. Int. J. Pharm., 124:97-105, 1995.

[10]. H.J. Robson, D.Q.M. Craig, D. Deutsch, An investigation into the release of cefuroxime axetil from taste-masked stearic acid microspheres Part 1: The influence of the dissolution medium on the drug release profile and the physical integrity of the microspheres. Int. J. Pharm., 190:183-192, 1999.

[11]. H.J. Robson, D.Q.M. Craig, D. Deutsch, An investigation into the release of cefuroxime axetil from taste-masked stearic acid microspheres Part 2: The effects of buffer composition on drug release. Int. J. Pharm., 195:137-145, 2000.

[12]. H.J. Robson, D.Q.M. Craig, D. Deutsch, An investigation into the release of cefuroxime axetil from taste-masked stearic acid microspheres Part 3: The use of DSC and HSDSC as means of characterising the interaction of the microspheres with buffered media. Int. J. Pharm., 201:211-219, 2000.

[13]. A.P. Rokhade, S.A. Agnihotri, S.A. Patil, N.N. Mallikarjuna, P.V. Kulkarni, T.M. Aminabhavi, Semi-interpenetrating polymer network microspheres of gelatin and sodium carboxymethyl cellulose for controlled release of ketorolac tromethamine. Carbohydr. Polymers, 65:243-252, 2006.

[14]. L.P. Wong, C.A. Gilligan, L.W. Po, Preparation and characterisation of sustained release ibuprofen-cetostearyl alcohol spheres. Int. J. Pharm., 83:95-114, 1992.

[15]. S. Geresh, G.Y. Gdalevsky, I. Gilboa, J. Voorspoels, J.P. Remon, J. Kost, Bioadhesive grafted starch copolymers as platforms for peroral drug delivery: a study of theophylline release. J. Control. Release, 94:391-399, 2004.

[16]. J.L. Ford, K. Mitchell, P. Rowe, D.J. Armstrong, P.N.C. Elliott, C. Rostron, J.E. Hogan, Mathematical modelling of drug release from hydroxypropylmethylcellulose matricies: effect of temperature. Int. J. Pharm., 71:95-104, 1991.

[17]. K. Gjellan, A. Magnusson, R. Ahlgren, K. Callmer, D. Christensen, U. Espmarker, L. Jacobsen, K. Jarring, G. Lundin, G. Nilsson, J. Waltersson, A collaborative study of the in vitro dissolution of acetylsalicylic acid gastroresistant capsules comparing the flow-through cell method with the USP paddle method. Int. J. Pharm., 151:81-90, 1997.

[18]. M.D. Likar, H.L. Mansour, J.W. Harwood, Development and validation of a dissolution test for a once-a-day combination tablet of immediate-release cetirizine dihydrochloride and extended-release pseudoephedrine hydrochloride. J. Pharm. Biomed. Analysis, 39:543-551, 2005.

[19]. G.M. Khan, J.B. Zhu, Ibuprofen release kinetics from controlled-release tablets granulated with aqueous polymeric dispersion of ethylcellulose II: Influence of several parameters and coexcipients. J. Control. Release, 56:127-134, 1998.

[20]. M.A. El-Massik, I.A. Darwish, E.E. Hassan, L.K. El-Khordagui, Development of a dissolution medium for glibenclamide. Int. J. Pharm., 140:69-76, 1996.

[21]. O.I. Corrigan, Y. Devlin, J. Butler, Influence of dissolution medium buffer composition on ketoprofen release from ER products and in 
vitro-in vivo correlation. Int. J. Pharm., 254:147-154, 2003.

[22]. K.A. Levis, M.E. Lane, O.I. Corrigan, Effect of buffer media composition on the solubility and effective permeability coefficient of ibuprofen. Int. J. Pharm., 253:49-59, 2003.

[23]. C.A. Kagadis, N.H. Choulis, Ibuprofen microcapsules from stearic acid. Part One:
Effect of particle size. Pharmazie, 40:807-808, 1985.

[24]. N.A. Peppas, J.J. Sahlin, A simple equation for the description of solute release III. Coupling of diffusion and relaxation. Int. J. Pharm., 57:169-172, 1989. 